\title{
Modelling and simulation of a Thrombectomy Probe applied to the Middle Cerebral Artery by using the Bond Graph technique
}

\author{
G. Romero, I. Higuera, J. Félez \\ Higher Technical School of Industrial Engineering \\ Technical University of Madrid (Spain) \\ \{gregorio.romero, irene.higuera, jesus.felez\}@upm.es
}

Keywords: Biomedical engineering, Thrombectomy Devices, rheology.

\begin{abstract}
Thrombosis is produced by the formation of a clot inside blood vessels causing an abrupt interruption of the blood flow. In the cerebral arteries, this occlusion can take place due to the presence of a clot that has formed at another location of greater diameter. It then obstructs the cerebral artery due to its smaller cross section. The process concerning the removal of this obstruction involves catheterisation. The experimental probe under study in this paper was developed by Dr G. Pearce and Reverend Neil Perkinson [1]. The probe, which when developed further may form the basis of a new Thrombectomy Aspiration Device (TAD) is called the GPTAD. Once fully developed, the GPTAD may provide a means of clot removal from vessels in the human arterial system e.g. the cerebral vessels. The modelling that we present in this paper, taking into account the catheter, the probe, artery, blood clot and adhesion forces, may assist with the optimisation of the design of the GPTAD probe . In the model used for the simulation both mechanical and hydraulic aspects have been considered with the purpose of combining the effect of the fluid-blood transmission for the different sections of the vein and the catheter.
\end{abstract}

\section{INTRODUCTION}

Stroke is a major cause of morbidity and mortality world-wide. In the UK there are 130,000 strokes each year [2]. Even patients who survive the initial insult of such a cerebral vascular event are often left with residual disability resulting in profound impact on their life and life expectancy. Approximately $85 \%$ of strokes are caused by a blood clot. Intravenous thrombolysis using alteplase is an effective treatment for acute stroke [3]. However, there are limitations on its use. Due to the risk of hemorrhage it is contraindicated in patients who have undergone recent surgery. In addition, it has to be given at a relatively early time after the stroke has occurred, and it is only currently licensed for use up to 3 hours post stroke [4]. A more recent study has indicated that it could possibly be used up to 4 to

\author{
G. Pearce, N.D. Perkinson \\ Biomedical Sciences Division \\ Wolverhampton University (United Kingdom) \\ gillpearce@googlemail.com, neil@perkinson.org.uk
}

5 hours post-stroke. It can therefore only currently be used in particular patients who have suffered a stroke.

During the development of potential new medical devices computer pre-modelling may be required to help in the optimization and fine-tuning of the devices.

In the case of a device based on the GPTAD probe, the extraction of the obstructing element (i.e. the blood clot) by the GPTAD would be done by means of vacuum suction provided by the action of a pump represented in the model as an effort source. To do this, an increasing pressure would be initially considered over time until it took on some values that are ideal for generating the suction required to remove the clot. Later a section of flexible tube will be considered and the different phenomena which take place inside this tube will be modelled: In the first place, linear load loss will be represented caused by the friction of the fluid particles with the walls of the tube. Secondly, the fluid's own inertia will be taken into account as its movement must be overcome. And finally, the compressibility of the blood on being subjected to a pressure figure under the conditions of that circulation section will be taken into account. In the final part of this tube and before the artery the so-called 'GPTAD' probe would be positioned. It creates the suction vortex when changing cross section between the tube and the artery and, therefore, the blood circulation speed is modified. This phenomenon is represented as a local load loss. Then, and until the clot has been located, the artery will be considered as another tube section in which the same phenomena as in the tube section corresponding to the catheter will be found. The difference to keep in mind in this second tube section is that artery elasticity will need to be taken into account, not only crosswise but also lengthwise by means of compliances. Finally the clot will be located at the other end of this artery. To be able to study in more detail what happens when this obstacle is sucked away and to facilitate the study of the results, the domain will be changed from hydraulics to mechanics. Therefore, the force required to separate the clot from the arterial wall will be analysed by modelling a compliance-resistance system to represent the interaction between both parts. A viscous damper will be used that allows considering the change from static to dynamic friction in its relative movement. 
To implement the phenomena by defining the different sections of the mechanism studied in the paper, blood is deemed to be an incompressible fluid, while the tube between the pump and the 'GPTAD' probe is deemed to be not elastic. The loss load will be due mainly to the existent flow, which by means of previous studies has been shown to be laminar.

The main objective of the development of this simulation model is to obtain the minimum pressure required to perform the extraction and to check that this pressure as well as the time required to complete the operation are reasonable for possible potential use on a patient and are similar to experimentally obtained data. In addition, it must be checked that that the cerebral arteries are able to support the force to which they are subjected, as well as the components comprising the proposed device, based on the 'GPTAD' probe, and that the blood flow generated acquires speeds that fall within admissible parameters. These studies and confirmations may help in optimising the probe and its possible future use in patients with thrombosis.

This paper gives a brief description of the proposed device, based on the 'GPTAD' probe, under study, as well as the parts comprising it. Next, the model used for the simulation is described as well as the phenomena considered, and, in addition, the values of the parameters used are defined. Lastly the results obtained and the conclusions of this study are attached.

\section{2. 'GP' TAD - A POTETIAL THROMBUS ASPIRATION DEVICE}

The proposed method for using the 'GPTAD' (figure 1) [1] would involve using a vacuum pump to provide the necessary suction pressure for the operation, joined to a very long catheter; with the 'GPTAD' probe located at the end of this catheter. The probe would be introduced into an artery in close proximity to the occluding blood clot, and be positioned at a distance of approximately $3 \mathrm{~mm}$ from it. Then the suction would begin until the clot was extracted. The clot would cross the $3 \mathrm{~mm}$ that separated it from the 'GPTAD' probe and when clot capture occured the 'GPTAD' would be removed from the body.

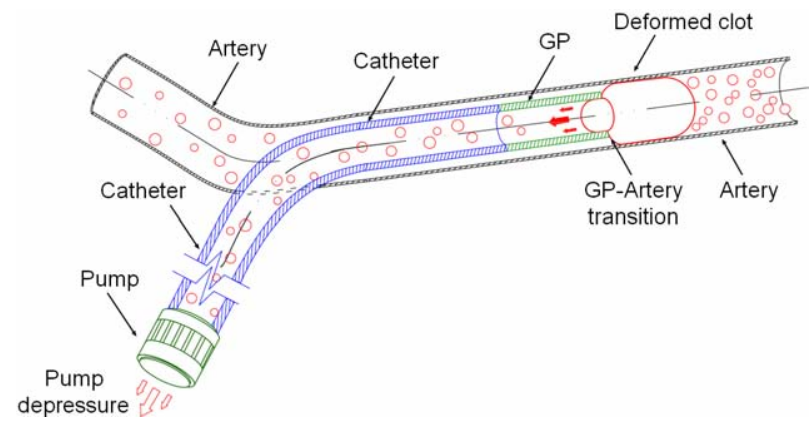

Figure 1. Schematic view of the GPTAD
The 'GPTAD' is currently being developed as a potential thrombus aspiration device through a series of in vitro studies. The probe has the potential to be used in relatively small arteries. It has no moving parts and therefore should reduce the risk of breakage in a vessel. Since it does not touch the clot itself it should also reduce the risk of clot disruption and downstream embolisation. Thrombectomy is achieved by aspiration through a catheter in which the 'GPTAD' probe is embedded. The internal surface has been mathematically developed. It is also associated with low forces at the periphery of the probe which may therefore reduce the risk of arterial collapse during aspiration of the clot $[5,6,7]$.

In addition, different existing techniques have been considered for the modelling and simulation [9]. Some methods which have been considered for their applicability are the Boltzmann flow simulation technique, and finite elements modelling and its implementation in Matlab (C) software, with 2D or 3D models; or by means of Laplace transformations using Dynamic Motion Solver (C) software. Mainly, these studies has served to analyze the distribution of the flow into the 'GPTAD' probe and to assist with its design to help obtain the necessary pressure just at the end according with in-vitro results.

The objective of this work is to introduce a onedimensional method to reproduce the entire model (pump, catheter, probe, artery, blood clot and adhesion forces) and it can be used in showing the potential performance of the GPTAD probe under different conditions of blood flow, size of blood clot, in a given vessel. The results (pressures vs times) in different blood clot situations are new - only preliminary results from in-vitro experiments in some of the situations have been obtained - and the modelling here shown has not been applied entirely to the 'GPTAD' probe previously.

The method chosen for the representation and simulation of this model is the Bond Graph technique [10]. Some medical models has been modelled by using this technique [11, 12] and its choice is based on the fact that this technique allows assimilating the model to an electric circuit made up of resistances, capacitances and inductances. Therefore, it is possible to obtain the results in a simple way by evaluating flows and efforts that join and connect the components of the model. To obtain the simulation of the model, Bondin (C) software has been used [13]. This program allows obtaining the evolution of the characteristic parameters of the model as well as letting them be compared.

\section{MODELLING PUMP, CATHETER AND PROBE}

First the pump, which is the component that creates the necessary pressure to carry out the extraction, is positioned. It can represented by a variable pressure source $(\mathrm{Se})$ whose value will increase from zero to a non-determined value 
suitable for carrying out this operation and will be obtained from the optimization of the developed model. The time taken to reach the maximum value of pressure has been obtained from experience and must be about $3 \mathrm{sec}$., after which time the pressure provided by the pump remains constant.

After the pump is the catheter, a $110 \mathrm{~cm}$ long $1 \mathrm{~mm}$ diameter hollow cylindrical tube which is joined to the 'GPTAD' probe of the same diameter and a length of 20 $\mathrm{mm}$. In order to represent both elements, they are considered as several pipe sections bearing in mind the different phenomena that take place in their interior: load and inertia loss, and fluid compressibility [14]. Due to the length of the catheter, it can be partitioned in identical sections (ten sections) and it can be represented by ten sub-models that include the three following described phenomena. Strictly, this partition is not necessary to do due to the linear behaviour into the catheter, but it'll helps to study better the existent pressure in each section; thanks to this representation, it will be possible to study the evolution of the pressure loss along the catheter (fig. 9).

Firstly, the flow inertia to be overcome in its movement is taken into account and considering a section with circular geometry it must be represented by a type I port and a type 1 junction and can be modelled with this expression:

$$
I=\frac{\rho \cdot L}{\pi \cdot(D / 2)^{2}}
$$

where $\rho$ is the blood density, $\mathrm{L}$ the length of the pipe section and $\mathrm{D}$ its diameter.

The blood has been deemed to be an incompressible fluid; if we wanted to take into account the blood compressibility, it should be included due to it acts as a compliance producing a decrease in volume when the pressure required for compression is increased. This behaviour would be dependent on Bulk's blood coefficient (B) and it could be defined as a type 0 junction and a capacitance of $K$ value (in our study case it should be eliminated in figures 2 and 3):

$$
K=4 \cdot B / \pi \cdot D^{2} \cdot L
$$

Lastly, due to the friction between the liquid particles and the pipe walls, appear load losses. Due to these being straight pipes, only linear load losses are taken into account. As this pipe is horizontal and of constant cross section in each section, the load loss is reduced to a pressure loss as the fluid advances along the pipe, the loss being progressive and proportional to the length of the pipe. This pressure losses can be represented by a resistance and a type 1 junction, and if we assume when artery is $100 \%$ occluded there is no flow and Reynolds number is only significant if turbulent flow is involved, the equation that governs its behaviour can be determined by following expression (3), where $\eta$ is the dynamic viscosity of the blood flow, $L$ the length of the pipe section and $\mathrm{D}$ its diameter.

$$
R=\frac{128 \cdot \eta \cdot L}{\pi \cdot D^{4}}
$$

The flow of blood may well be turbulent at an arterial bifurcation for example, or in the case of an atheromatous plaque associated with a pathological blood clot, not existent in this case. In these situations it would be necessary to determine the load losses for turbulent flow and taking into account Reynolds number.

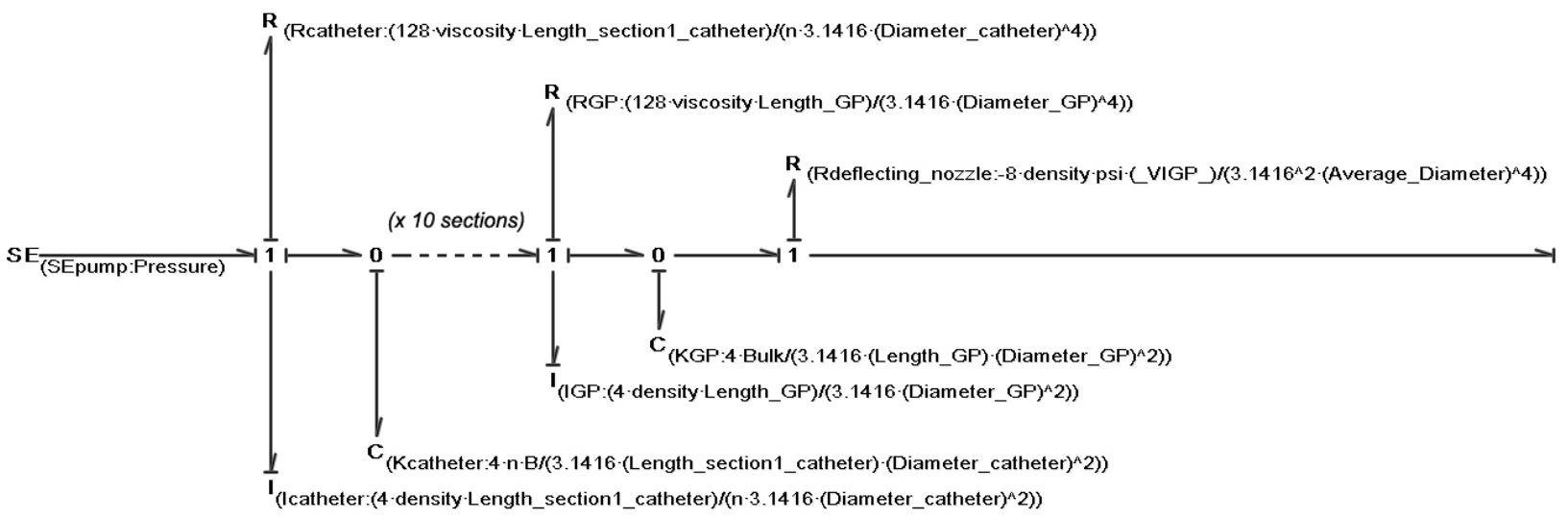

Figure 2. Modelling the pump, catheter and 'GPTAD' probe components by Bond Graph technique

Later the 'GPTAD' probe (see figure 1) must be positioned and it can be represented by the same three previous phenomena and with the corresponding values. In addition, due to the artery being located at the end of the 
'GPTAD' probe, it is necessary to consider the transition between both elements as a secondary load loss caused by the difference in diameter of the 'GPTAD' probe and the artery respectively and the subsequent variations in flow. These load losses can be represented as a resistance and can be calculated with the following expression (4), where $\rho$ is the blood density, $\mathrm{Q}$ is the flow which circulates in the section between the end of the 'GPTAD' probe and the artery, and the diameter $\mathrm{D}$ is the mean value between the probe $\left(D_{\text {probe }}\right)$ and the artery $\left(D_{\text {artery }}\right)$.

$$
R=8 \cdot \rho \cdot \xi \cdot \frac{Q}{\pi^{2} \cdot D^{4}}
$$

The load loss coefficient $\xi$ is a dimensionless parameter that quantifies the loss produced and depends on the geometry of the frustro-conical aperture; since this is a narrowing, this value is 0.4 . The flow $\mathrm{Q}$ is not constant during the extraction, so to calculate the value into the model according to expression (4), its value at each instant can be considered as the flow of the inertance 'IGP' that represents the 'GPTAD' probe, represented by '_VIGP_' in figure 2.

\section{MODELLING THE ARTERY AND TRANSITION TO CLOT DESCRIPTION}

The artery is located between the end of the 'GPTAD' probe and the clot. It can be included in the model as another section of a pipe, similar to the catheter and the 'GPTAD' probe and it must be defined by the loss of linear load (R), the inertia (I) and the compressibility of the blood (C) (figure 3). In addition, it is necessary to insert a parameter that represents the compressibility of the artery, in line with its Young's modulus:

$$
K=\frac{E \cdot h}{V_{0} \cdot 2 \cdot r_{0}}
$$

where $\mathrm{E}$ is its Young's modulus, $\mathrm{h}$ is the thickness of the artery, $\mathrm{V}_{0}$ is the artery initial volume and $\mathrm{r}_{0}$ is the artery initial radius.

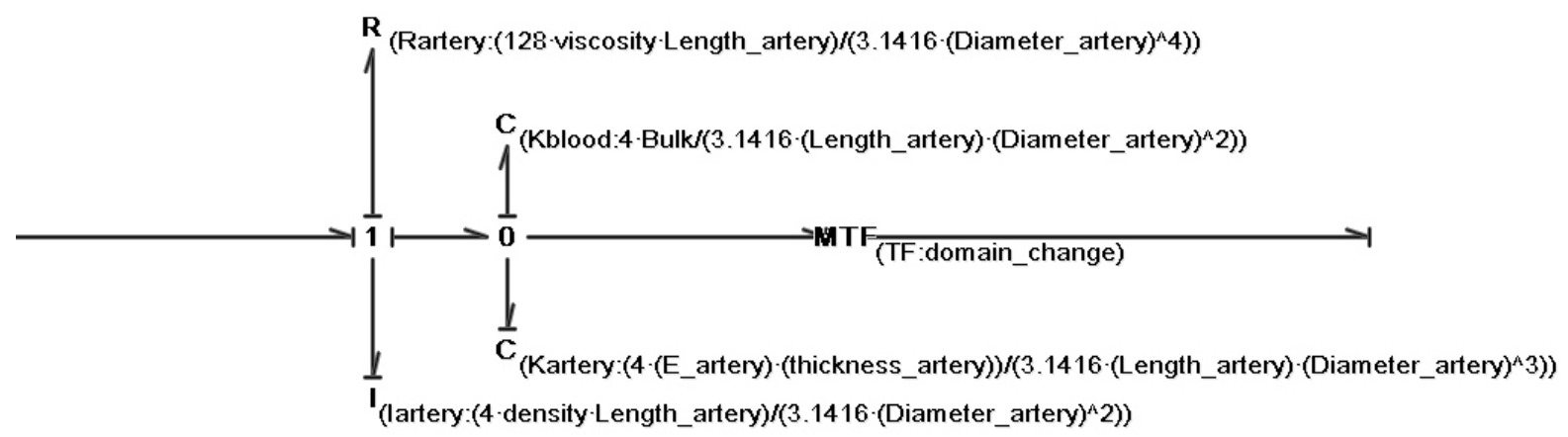

Figure 3. Modelling the artery and transition to clot by Bond Graph technique.

Once the elements corresponding to pump, catheter, GP ATD device and artery have been defined by fluid mechanics, it is necessary to change from the domain of hydraulics to mechanics, to be able to evaluate the movements and efforts in the clot, as well as to define the physical friction between the clot and the artery. This domain change is carried out by a Transformer (TF) element. To calculate the value of the coefficient defining this element, the change in the definition of the flow before and after this element is evaluated; it's known that there is no leakage around the clot and no blood gets passed the clot when it is causing $100 \%$ occlusion of an artery. The coefficient must be determined by evaluating the required change between both domains; before the TF element, the flow is in the hydraulics domain, while afterwards, it is in the mechanics domain. Then, the coefficient of the TF element must be the inverse of the section of artery.

\section{MODELLING THE CLOT}

Accurately defining the clot model in order to model it is the most complex part of the model. A clot is a cylindrically-shaped element of [3-5] cm long, and of a mass that falls between [0.5-1] gram. This element has usually formed in another location, usually in a vascular artery, and has become dislodged remaining trapped in smaller diameter arteries, such as the cerebral artery considered in this work. Therefore, due to this difference in diameter, the clot becomes attached to the wall by a force that needs to be overcome in order to begin its movement for removal. In addition, the relative movement between the clot and the artery presents static and dynamic friction, which needs to be taken into account. If a correct approximation to reality is to be achieved all these phenomena, as well as the circumstances restricting clot movement, need to be considered. 
As already stated, the phenomenon preventing clot movement is the difference in diameter between the clot and the artery where it is located. Experimental data indicate that the clot begins its movement when the extraction force is equal to $0.01 \mathrm{~N}$.

In order to decide when to begin the movement of the obstructive element, firstly we need to insert a compliance into the model to measure the force supported by the beginning of the clot and its deformation.

To obtain the value of this compliance the phenomenon of surface tension must be taken into account, since it is this that joins the clot to the artery. This surface tension $\gamma$ comes about from the attraction forces between molecules and is defined as force by unit length l. If we take a sphere into the artery, the surface tension would act on the circumference of the contact between the clot and the artery. If we take into account the value of the length of contact from the radius $r$ of the artery, the value for the surface tension can be obtained and, in turn, the value for the compliance coefficient as:

$$
K=\gamma=F / l=1,061 \mathrm{~N} / \mathrm{m}
$$

As stated above, the clot is between [3-5] cm long, which means it can be broken down into the union of several spheres, all with the same constant. Since over the whole surface of the clot there are adhesion forces, to obtain a correct approximation it is necessary to consider the existence of a sphere for every $0.1 \mathrm{~mm}$. This means that between 300 and 500 spheres would need to be included in the model. On the other hand, all the spheres must be located in parallel to obtain the resultant adhesion force, and since all the individual compliances are equal, the equivalent compliance for a $5 \mathrm{~cm}$ clot can be had from the form appearing in the following expression:

$$
K_{e q}=\frac{K}{n}=\frac{1,061}{500}=0,002122 \mathrm{~N} / \mathrm{m}
$$

To know when the force will be reached in this equivalent compliance and, therefore, when the clot movement will begin, it is essential to calculate the displacement of the compliance when it is subjected to 0.01 $\mathrm{N}$ through a typical compliance equation. Therefore, only when the compliance undergoes this displacement should the clot be allowed to move; to the contrary it would be prevented.

$$
x=\frac{F}{K_{e q}}=\frac{0,01 N}{0,002122 N / m}=4,7124 m(8)
$$

Secondly it is necessary to insert the resistance that represents the friction between the clot and the arterial wall.
The value of this parameter must be variable depending on whether the clot has not begun its movement (static friction) or if it is already in movement (dynamic friction), so that when the clot begins to move the friction value will drop considerably. This value is obtained starting from the Stokes equation and can be given a value of $2.5 \cdot 10^{-6} \mathrm{~N} \cdot \mathrm{s} / \mathrm{m}$ for the static friction and an order of magnitude lower than for the dynamic friction. The transition between both values marks the beginning or end of the clot movement by means of the displacement of the compliance representing the deformation described in expression (8). So, when the displacement undergone by this compliance is less than that calculated, static friction will rule; to the contrary, if it is greater, the friction will be dynamic.

In addition to the compliance and the resistance inserted, the model must have an inertance that represents the mass of the clot ( 1gr) (figure 5).

\subsection{Clot suction}

To ensure that the clot remains at rest while the force existing at its beginning is less than $0.01 \mathrm{~N}$, a compliance-resistance system joined to a wall (zero flow source) must be used (figure 5). In this system, while the clot does not receive the force of minimum suction, it has zero speed. However, when it begins its movement, the compliance-resistance system must be cancelled allowing its extraction.

Therefore, while the force representing the deformation of the clot (represented by 'Kclot' in figure 5) is lower than $0.01 \mathrm{~N}$, it will remain attached to the wall, thereby preventing any movement. To the contrary, if the force exceeds this figure the model will cause the bond imposed by the compliance-resistance system to be eliminated with the clot becoming free and moving in accordance with the suction pressure acting on it from the system, letting it be removed.

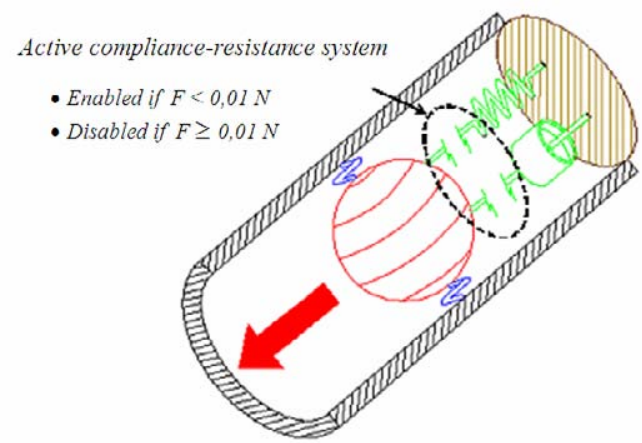

Figure 4. Compliance-resistance system.

Therefore, the elimination of the compliance-resistance system, initially of $1 \cdot 10^{10} \mathrm{~N} / \mathrm{m}$ and $1 \cdot 10^{9} \mathrm{~N} \cdot \mathrm{s} / \mathrm{m}$ respectively, will be performed from the displacement of the compliance representing the clot deformation, as was seen in the condition imposed on the static/dynamic friction. 


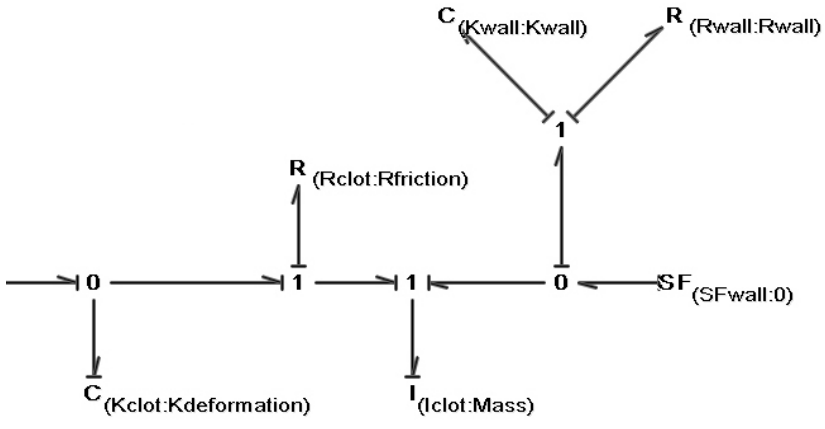

Figure 5. Modelling clot and suction by Bond Graph tech.

The implementation of the model required for the simulation has been made connecting the different components show previously (figures 2, 3 and 5). The full model has been presented divided into three ordered blocks. The fundamental object of this paper consists in determining and optimizing the minimum pressure required for the extraction of a blood clot depending on the clot size.

\section{RESULTS}

To do the correct simulation and optimization, by varying the values of the pressure source and clot length, the movement of the clot and the time required for its extraction are measured, thereby obtaining the optimum minimum pressure. To carry out the model validation, the values of the parameters used in the simulation are listed in following table.

\begin{tabular}{|l|c|}
\hline Pressure & $0-[-30,-60] \mathrm{kPa}$ \\
\hline Catheter length (L) & $1.10 \mathrm{~m}$ \\
\hline Catheter diameter (D) & $1 \cdot 10^{-3} \mathrm{~m}$ \\
\hline 'GP' length (L) & $20 \cdot 10^{-3} \mathrm{~m}$ \\
\hline 'GP' diameter (D) & $1 \cdot 10^{-3} \mathrm{~m}$ \\
\hline 'GP' thickness (h) & $1 \cdot 10^{-4} \mathrm{~m}$ \\
\hline 'GP'-artery mean diameter ( $\left.\mathrm{D}_{\mathrm{rn}}\right)$ & $2 \cdot 10^{-3} \mathrm{~m}$ \\
Artery thickness (h) & $1 \cdot 10^{-4} \mathrm{~m}$ \\
\hline Artery diameter (D) & $3 \cdot 10^{-3} \mathrm{~m}$ \\
\hline Adtery length $\left(\mathrm{L}_{\mathrm{a}}\right)$ & $3 \cdot 10^{-3} \mathrm{~m}$ \\
\hline
\end{tabular}

\begin{tabular}{|c|c|}
\hline Artery Young modulus (E) & $2.8 \cdot 10^{9} \mathrm{~N} / \mathrm{m}$ \\
\hline Load loss coefficient ( $\xi)$ & 0.4 [no dim.] \\
\hline Domain change coef. (r) & $14.147 \cdot 10^{4}[\mathrm{n} \circ \mathrm{dim}]$. \\
\hline Bulk's coefficient (B) & $2.2 \cdot 10^{9} \mathrm{~N} / \mathrm{m}$ \\
\hline Blood Viscosity (१) & $0.0035 \mathrm{~Pa}$ 's \\
\hline Blood Density $(\rho)$ & $1,060 \mathrm{~kg} / \mathrm{m}^{3}$ \\
\hline Static friction & $2.5 \cdot 10^{-6} \mathrm{~N} \cdot \mathrm{d} / \mathrm{m}$ \\
\hline Dynamic friction & $2.5 \cdot 10^{-8} \mathrm{~N} \cdot \mathrm{s} / \mathrm{m}$ \\
\hline Clot weight & $0.001 \mathrm{~kg}$ \\
\hline
\end{tabular}

Table 1. Parameter values

Likewise, by introducing gauges into the model, pressure loss must be evaluated through the sections determining where these losses are concentrated. Also evaluated is if the artery possesses the necessary strength to support the pressure to which it is subjected during the proposed procedure.

The results of these parameters have been evaluated for the sizes of the clots of $5 \mathrm{~cm}$ and $3 \mathrm{~cm}$. For each pressure and size of the clot, the necessary times to reach the necessary force at the end of the clot will be obtained so that its movement begins, and is picked up, as well as the time that the total extraction takes.
In the following figure the final time to end clot movement and the extraction obtained for both sizes are shown.

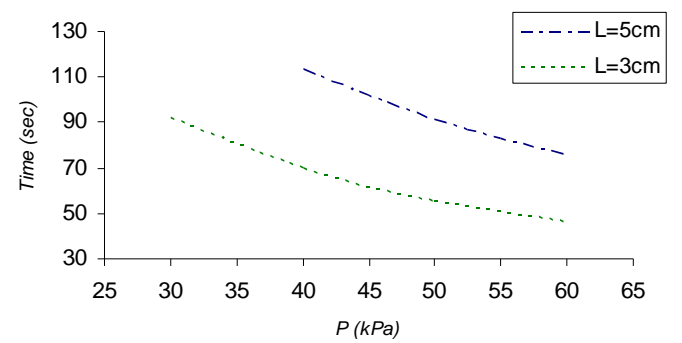

Figure 6. Final time to end the extraction with different sizes and suction pressures.

It is observed that for a longer length of clot, more time is needed with the same pressure to reach the necessary force so that the movement begins and for the subsequent extraction. Also, for the same size of the obstructive element, when the suction pressure increases, the time needed to complete the operation diminishes. These results are coherent with what would need to be obtained.

It is necessary to highlight that for a size of $5 \mathrm{~cm}$ long and a pressure of $30 \mathrm{kPa}$, no time value is attached; no movement of the clot can be appreciated for any time when using this suction pressure in the simulation due to the fact that pressure is insufficient to be able to create a force of $0.01 \mathrm{~N}$ before the obstructive element, therefore it remains indefinitely at rest.

One of the conclusions looking at the simulations of this model is that it can be appreciated that for a length of $5 \mathrm{~cm}$ the necessary time for the extraction is in the range of $[80,120]$ seconds; in addition we can see how we could use any pressure but $40 \mathrm{kPa}$ is enough. However, for a length of $3 \mathrm{~cm}$ it is observed that the greater the pressure, the faster the clot moves; so with a pressure of between 30 and $40 \mathrm{kPa}$ the clot can be extracted.

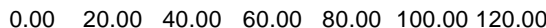

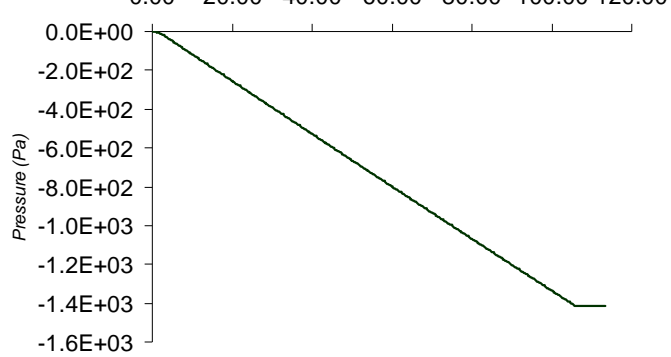

Figure 7. Clot pressure at beginning of movement.

Using a $40 \mathrm{kPa}$ suction pressure and considering a $5 \mathrm{~cm}$ long obstructive clot of 1gr. mass, in figure 7 the evolution of the suction pressure supported by the clot can be observed. It can be seen that it undergoes an increase over 
time until it reaches a value of $1.41 \mathrm{kPa}$ at 112 seconds. This pressure corresponds to $0.01 \mathrm{~N}$, the point at which the clot starts its movement, and it must be lower than the cerebral arteries are able to support.

Following figure shows clot movement. It can be seen that its movement is zero until 112 seconds, a point at which it reaches a force of $0.01 \mathrm{~N}$. From this instant it begins its movement. The clot must travel $3 \mathrm{~mm}$ through the artery, which is the distance that separates it from the end of the 'GPTAD' probe, in which the clot will be captured. It can be observed that the clot needs less than 2 seconds. to travel this distance, giving a total time of $\sim 114$ seconds.

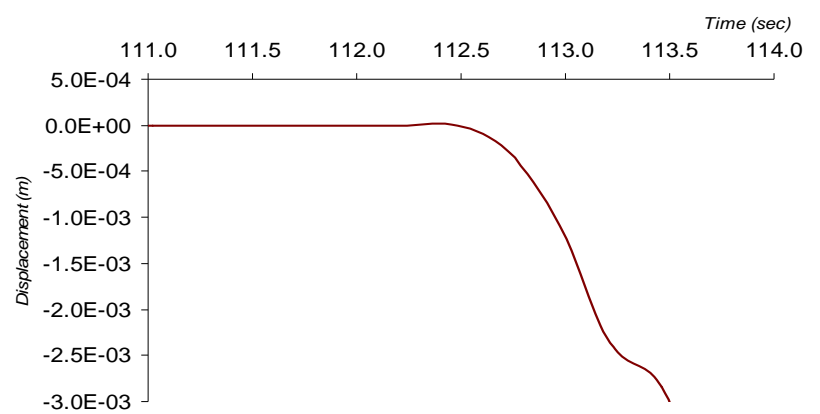

Figure 8. Blood clot movement.

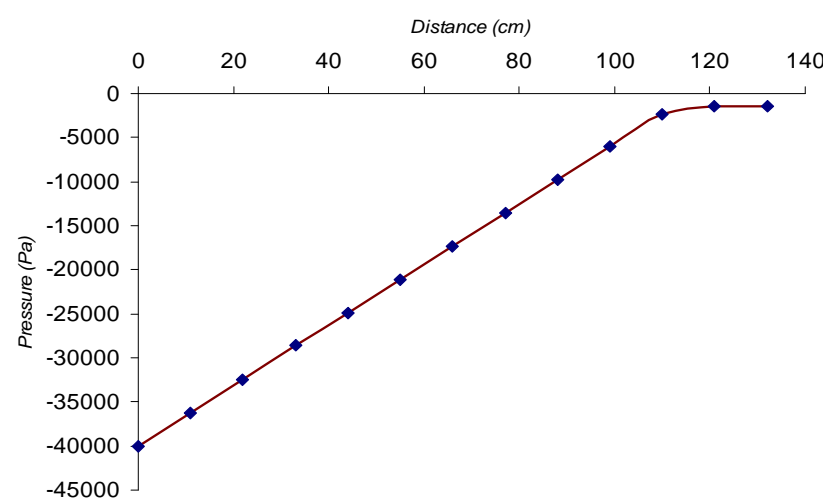

Figure 9. Loss in pressure in the catheter, GP and artery.

Finally, in figure 9 the loss in pressure is shown from the pump up to the clot for a length of $5 \mathrm{~cm}$ and a suction pressure of $40 \mathrm{kPa}$. The proportionality of the loss in pressure is observed in the catheter (distance $0-110 \mathrm{~cm}$ ) and its greater magnitude in respect of the loss experienced in the 'GPTAD' probe and artery. It has been obtained when the clot was moving and it can be seen that the loss of pressure appears mainly in the catheter part due to the length of this component and also how the loss of pressure is the same in each division. After this loss of pressure, the pressure is 99\% stabilized in the 'GPTAD' probe (the loss of pressure is lower than in the catheter). Finally a small percentage drop of the pressure removes the clot.

\section{CONCLUSIONS}

Experimental data indicate that the extraction of blood clots can take place in an interval of time of [60 - 120] sec. In figure 6,7 and 8 it can be seen that the simulation produces the extraction in 92 or 114 sec., a figure that is coherent with experience.

The developed model indicates that the minimum value of pressure for the extraction of a $5 \mathrm{~cm}$ long 1 gr. clot is 40 $\mathrm{kPa}$, while a pressure of $30 \mathrm{kPa}$ is enough for the suction of a $3 \mathrm{~cm}$ length.

On the other hand, studies demonstrate that the artery has a resistance of $750 \mathrm{mmHg}$, which is equivalent to 100 Kpa. This has been analyzed in the model and the pressure in this area rises to $1.41 \mathrm{kPa}$ (fig. 7), giving a very wide safety margin for the artery.

Finally it is shown that an important pressure loss takes place in the catheter joining the pump to the 'GPTAD' probe.The values obtained could possibly be used to optimize the geometry of any device developed based on the GPTAD probe, although to be really effective in an future optimization of the GPTAD probe, ways of representing the unique design features in the GPTAD probe must be incorporated into any model.

This work is a first attempt to simulate the operation of a proposed device based on the 'GPTAD' probe, assisting in its future applicability and compatibility with the experimental data obtained. The subsequent lines of work should focus on developing a highly accurate model to study, including modelling of the rechanneling of the blood flow after clot removal.

\section{FUTURE WORKS}

Additional simulations over a modified and adapted model has shown necessary pressures and times to remove clots of differents sizes during Peripheral Vascular Disease. No in-vitro data currently exists for this device in respect of its use in the leg to remove blood clots that arise during Peripheral Vascular Disease, so no comparisons can yet be made between in-vitro measurements using models and our Bond graph modeling, which are being analyzed nowadays

The presented model is now being implemented to model and simulate the coronary vessels of the heart. Future analysis will involve the GPTAD probe in proximity to both athromatous plaques and blood clots, in coronary vessels which become blocked in myocardial infarction.

\section{REFERENCES}

[1] Pearce, G. and Perkinson, N.D. 2006. "Biomechanical Probe”. International Patent Corporate Treatise (WO2006120464) published 2006-11-16 ; European patent (EP1893195 (A2)) published 2008-03-05; Japanese patent (JP2008639924 (T)) Published 200811-20; Chinese patent (CN101208049 (A)) published 2008-06-25. 
[2] Stroke association website. <http://www.stroke.org.uk/ /document.rm?id=330 >. Last accessed 30 ${ }^{\text {th }}$ Dec. 2008.

[3] Guidelines for the early management of adults with ischemic stroke. 2007. Stroke. Vol. 38, pp. 80-84.

[4] TA122 Alteplase for the treatment of acute ischaemic stroke: guidance. 2007. National Institute for clinical Excellence.

[5] Pearce, G. et al. 2007. "A new device for the treatment of thromboembolic strokes". Journal of Stroke and Cerebrovascular Diseases. Vol. 16, No. 4), pp. 167-172.

[6] Pearce, G. et al. 2008. "Modelling of the 'GP' Mechanical Thrombectomy Device MTD”. 10th International Conference on Computer Modeling and Simulation, pp. 499-502. Cambridge, UK.

[7] Pearce, G. et al. 2009. "An Investigation of fluid flow through a modified design for the 'GP' device”. 11th International Conference on Computer Modelling and Simulation, pp. 191-195. Cambridge, UK.

[8] Pearce, G. et al. 2008. "The design, optimisation, and testing of a new mechanical clot retrieval device for use in vascular surgery”. International Journal of Engineering Simulation. Vol. 9, No. 2, pp. 10-26.

[9] Rai, M. et al. 2009. "A Versatile Low cost Arterial Simulator”. 11th International Conference on Computer Modelling and Simulation, pp.196-199. Cambridge, UK.

[10] Karnopp, D.C. et al. 1990. "System Dynamics: A Unified Approach”. John Wiley \& Sons, Inc., Second edition.

[11] Zadpoor, A.A. et al. 2005. "A bond graph approach to the modelling of fluid-solid interaction in cardiovascular system's pulsatile flow”. 27th Annual International Conference of the IEEE in Medicine and Biology Society (EMBC05), Shanghai, China.

[12] Tabatabai, G.F. et al. 2005. "Spatiotemporal wavefront propagation in 3D geometric excitable heart tissue utilizing Bond Graph modelling technique”. 2005 International Conference on Bond Graph Modelling and Simulation (ICBGM'2005), New Orleans, USA.

[13] Romero, G. et al. 2009. "BONDIN: a new engineering simulation software for ODE and DAE systems with symbolic notation based on the Bond Graph technique”. 8th WSEAS Int. Conf. on Software engineering, parallel and distributed systems, pp. 90-97. Cambridge, UK.

[14] Margolis, D. 1979. "Bond graph fluid line models for inclusion with dynamic systems simulations”. Journal of the Franklin Institute. Vol. 308, No. 3, pp. 255-268. 